\title{
Gastric adenocarcinoma is concurrent with metastatic neuroendocrine cancer treated with nivolumab and chemotherapy: A case report
}

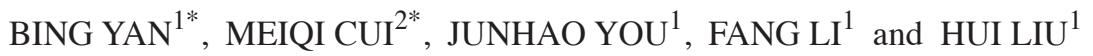 \\ Departments of ${ }^{1}$ Oncology and ${ }^{2}$ Outpatient, Hainan Branch of PLA General Hospital, Sanya, Hainan 572000, P.R. China
}

Received June 28, 2018; Accepted September 21, 2018

DOI: $10.3892 / \mathrm{mco} .2018 .1740$

\begin{abstract}
Gastric adenocarcinoma concurrent with metastatic neuroendocrine cancer (NEC) is rare. In the present case report, a 39-year-old male was first pathologically diagnosed by gastric endoscopy as having a highly differentiated adenocarcinoma. Next, positron emission tomography-computed tomography examination and bone marrow biopsy confirmed extensive metastasis. Subsequently, the patient underwent 6 cycles of immunotherapy (nivolumab, $160 \mathrm{mg}$ ) and 5 cycles of chemotherapy based on the XELOX regimen (oxaliplatin + capecitabine). Following this, the patient received the final cycles of nivolumab and XELOX; however, the patient then succumbed. Further biopsy of the metastatic collarbone lymph nodes indicated NEC. Overall, the progression-free survival was $\sim 3.5$ months, and overall survival (OS) was $\sim 6$ months. The case presented the possibility of concurrent gastric adenocarcinoma and NEC in the clinic. In addition, the efficacy of a combined regimen such as immunotherapy and chemotherapy for such disorders still requires further validation in the future.
\end{abstract}

\section{Introduction}

Although gastric cancer is thought to be a highly heterogeneous disease (1), adenocarcinoma is still the most frequent pathological type in clinics. Neuroendocrine cancer (NEC) in the stomach is uncommon and only accounts for approximately $0.1-0.2 \%$ of all cancers that occur in the organ (2). Concurrent occurrence of gastric adenocarcinoma and neuroendocrine cancer is rare and has only been registered in a few case reports so far.

Correspondence to: Dr Hui Liu, Department of Oncology, Hainan Branch of PLA General Hospital, 80 Jianglin Road, Sanya, Hainan 572000, P.R. China

E-mail: woshiliuhui1995@aliyun.com

\section{${ }^{*}$ Contributed equally}

Key words: gastric adenocarcinoma, neuroendocrine cancer, tumor markers, nivolumab, oxaliplatin + capecitabine
To date, such concurrent lesions have been classified into two subgroups according to their morphological features, named the composite-type and the collision-type; in the former, both elements seem to be mixed haphazardly, while in the latter, the tumors are considered double tumors with 'a hand in hand' conformation (3). Based on the complex relationship of the cancers, definite pathological diagnosis of such lesions is difficult. Most of the previous cases were diagnosed by gross specimens from surgery, such as gastrectomy. In 2010, the WHO named mixed adenoneuroendocrine carcinomas (MANECs), which present neuroendocrine cells (usually over $30 \%$ of all tumor cells) mixed with nonendocrine components (usually adenocarcinoma) (4), as a new category in the list of NECs. Interestingly, some of the previous cases are likely to be reclassified into this group retrospectively.

In this study, we present a case of gastric adenocarcinoma concurrent with metastatic NEC treated by nivolumab and chemotherapy (based on the XELOX regimen). The overall survival time of the patient was approximately 6 months. Our case addresses the possibility of concurrent gastric adenocarcinoma and NEC in the clinic; however, the efficacy of a combined regimen such as immunotherapy (nivolumab, for example) and chemotherapy for such disorders still needs further validation.

\section{Case report}

A 39 -year-old man was first revealed by the ${ }^{13} \mathrm{C}$ breath test to have an $H$. pylori infection during a routine physical examination; however, no treatment was adopted. Six months later, he suddenly presented tarry stool after drinking and underwent a gastric endoscopy, the pathological results of which indicated a well-differentiated adenocarcinoma on the gastric corpus (Fig. 1A); further immunohistochemical staining indicated the presence of CD4 (3+), CD8 (3+), MAGEA3 (2+), NY-ESO-1 (-), and PD-L1 (-) (Fig. 1B-F). Subsequent PET-CT examination showed the following: 1). Irregular wall thickening on the distal gastric corpus and antrum, particularly the greater curvature, indicated gastric cancer with adjacent fatty infiltration, and the greater omentum, ascending and transverse colon were likely to be involved; 2). Multiple lymph node metastases were present around the left supraclavicular and neck, to the right of the diaphragmatic feet, and in the left gastric artery area, celiac axis, liver and gastric ligament, small omental bursa, mesentery, retroperitoneal abdominal aorta and inferior vena cava; 3 ). The 

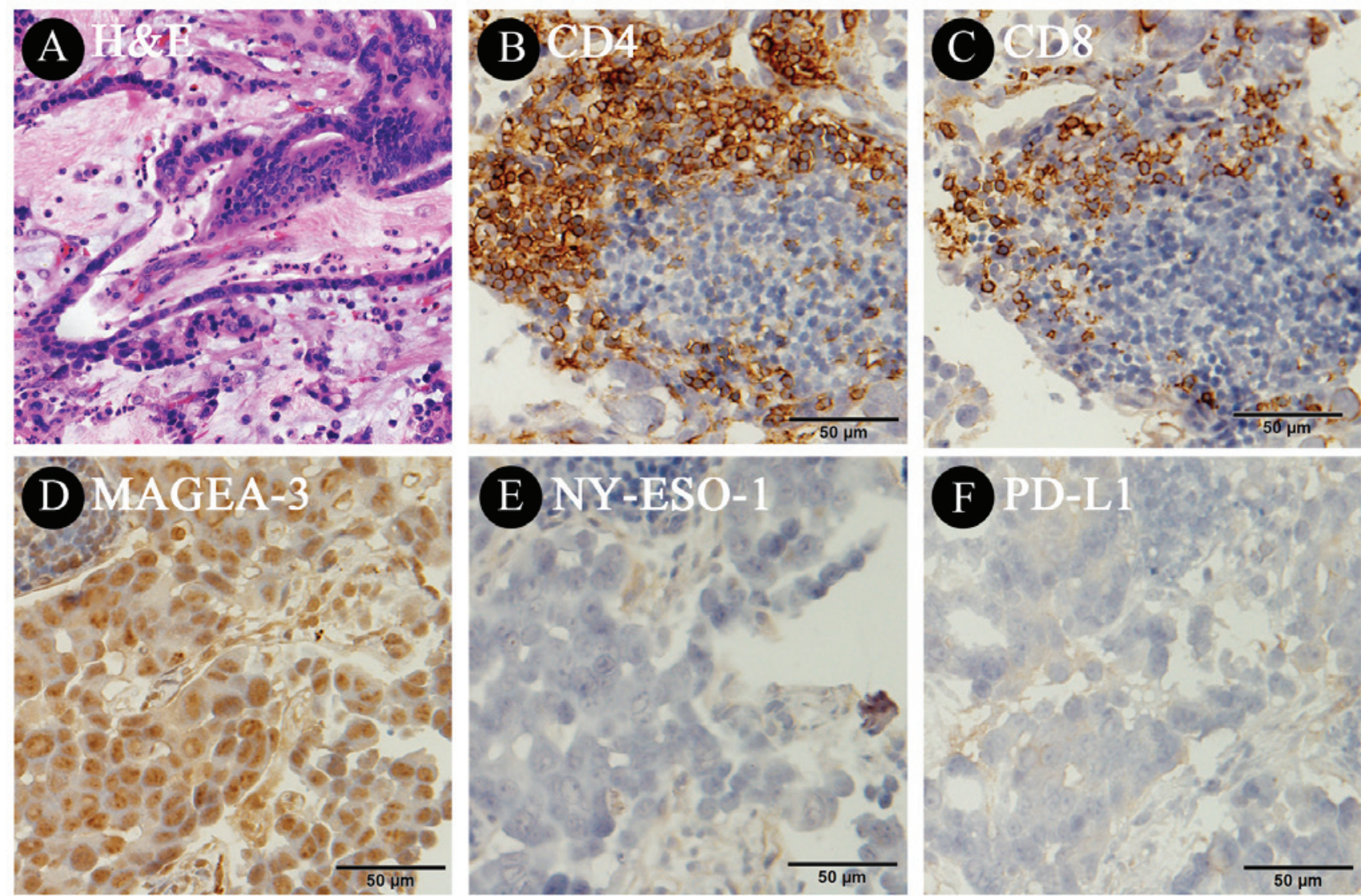

Figure 1. Histological results of H\&E staining and immunohistochemistry by gastric endoscopy. (A) Well-differentiated adenocarcinoma from the superficial mucous membrane layer of the gastric corpus (H\&E: magnification, x100). (B) CD4 membrane-positive cells clustered in the tumor (Magnification, x200). (C) CD8 membrane-positive cells could also be seen in the tumor, but the area was smaller than that observed for CD4 (magnification, x200). (D) MAGEA3 staining was diffusely positive in nearly all tumor cells. (E) NY-ESO-1 and (F) PD-L1 staining was negative in all tumor cells (magnification, x200). H\&E, hematoxylin and eosin; CD, cluster of differentiation; MAGEA3, melanoma antigen family member A3; NY-ESO-1, New York esophageal squamous cell carcinoma 1; PD-L1, programmed cell death 1 ligand 1.
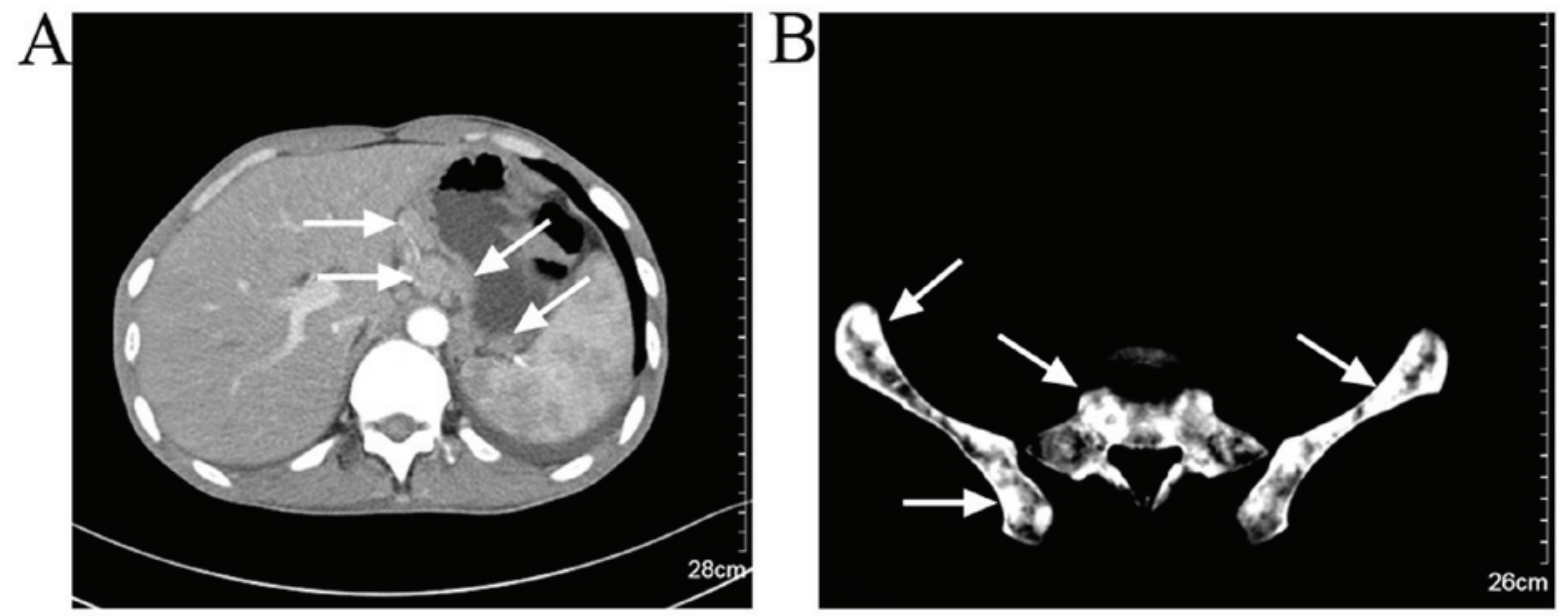

Figure 2. CT scan of the lesions previously reported by positron emission tomography-CT. (A) Abdominal enhanced CT indicates irregular wall thickening on the distal gastric corpus and antrum with heterogeneous enhancement, accompanied by multiple lymph node metastasis (white arrows). (B) Pelvic CT with bone window reveals extensive centrum and pelvic metastasis as well as marrow invasion (white arrows). CT, computed tomography.

right and left femoral cavity, as well as multiple bones throughout the body, presented metastatic lesions. The patient then underwent a bone marrow biopsy, which confirmed metastatic cancer, and FISH for Her-2 (negative). He then received the first cycle of nivolumab (160 mg) treatment in another hospital and came to our department. Further review of baseline images including chest, abdomen and pelvis computed tomography (CT) scans confirmed the previous imagological diagnosis (Fig. 2). Next, he 
received the second cycle of nivolumab and took capecitabine (1.5 g po twice per day, days 1-14 every 3 weeks) simultaneously. After that, another 3 cycles of nivolumab and XELOX (oxaliplatin $200 \mathrm{mg}$ ivgtt, day $1+$ capecitabine $1.5 \mathrm{~g}$ po twice per day, days 1-14) regimen treatments were executed; however, the intervals of the treatment plan were not executed as scheduled because of severe complications, including myelosuppression (grade 2-3 decreased platelet count and grade 1-2 anemia) and grade 1-3 hand-foot syndrome. Evaluation of the therapeutic effects was conducted by abdominal CT scan and a blood test for tumor markers as planned (Fig. 3). Stable disease (SD) and obvious progressive disease (PD) were detected after 3 and 5 cycles of treatment, respectively. He received the last 2 cycles of treatment even though the disease was considered to be PD. A further biopsy of the metastatic collarbone lymph nodes indicated neuroendocrine cancer with the following immunohistochemical staining: synaptophysin (+), CD56 (+), CK/CK7 (+), CK20 (-), Villin (-), Ki-67 (>75\%) (Fig. 4). All the treatments were then ceased because of the poor performance status and severe complications; he died on December 12. Written informed consent was obtained from the patient's father.

\section{Discussion}

Gastric adenocarcinoma concurrent with metastatic NEC is a rare phenomenon. To the best of our knowledge, concurrent gastric adenocarcinoma and NEC are uncommon and have only been registered in a few case reports (Table I) (5-23). In our study, the patient was treated with nivolumab and chemotherapy. Although a transient disease regression was observed, the PFS ( $\sim 3.5$ months) and OS ( $\sim 6$ months) were still unsatisfactory; the efficacy of the combined regimen for such disorders needs further validation in the future.

In recent years, the detection of a neuroendocrine element in gastrointestinal cancers has been increasingly registered, which has often prevented concise diagnosis. In fact, the majority of previous cases $(5,6,8-15,17-21,23)$ were diagnosed by gross specimens from surgeries such as gastrectomy because the neuroendocrine component is usually located in the mucosa, while the adenocarcinoma is seated in the deeper layers $(7,14,21,24)$; furthermore, each of the two cancer types can occasionally present pathological evidence for the differentiation of the other $(14,25-28)$ or the possibility of transformation from one type to the other (29). In our study, a MANEC (or, to be more precise, a high-grade MANEC by La Rosa's report) (30) likely occurred according to the new categorization by WHO in 2010 , considering that some reports have indicated that gastric NEC is prone to metastasis (13) and that the cells in metastatic sites are similar to those in the primary sites $(2,11)$. However, due to the lack of gross samples and autopsy, it is impossible to estimate the percentage of cell elements in the tumor, and thus, a definite diagnosis was difficult.

To date, consensus guidelines for the management of double or multiple original cancers have not been established. La Rosa et al (30) suggested that priority should be given to the more malignant elements in the mass. For our case, some guidelines recommended management in the same manner as for gastric adenocarcinomas (31). For example, Li et al (17) reported that the FOLFOX regimen in such cases could achieve a 12-month disease-free survival. However, as NEC

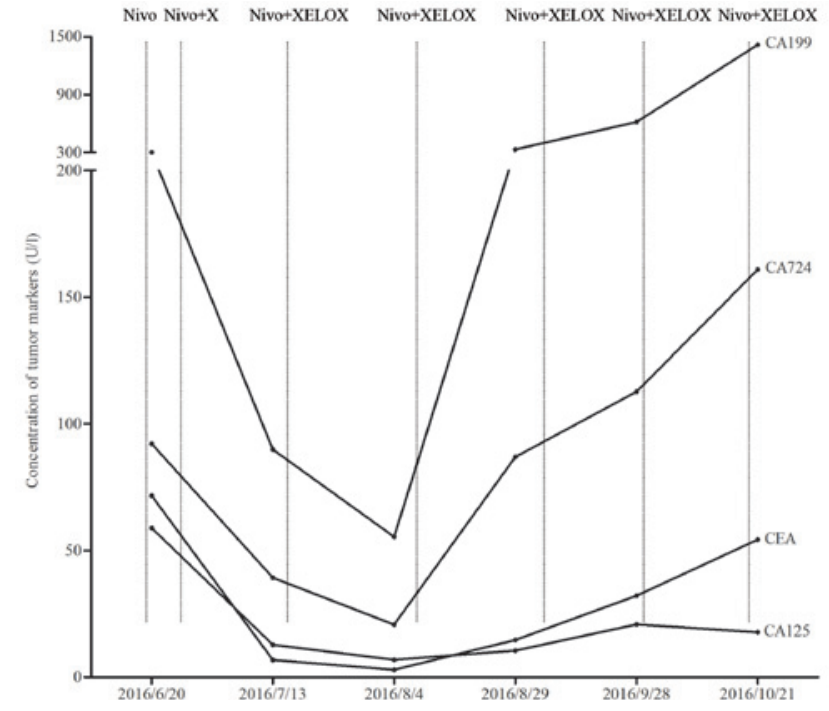

Figure 3. Variation in tumor markers during the course of treatment. Following 4 cycles of treatment, the levels of CA199, CA724, CEA and CA125 decreased markedly, and the image examination indicated a stable disease; however, an evident progressive disease could be detected following this, and CA199 and CA724 increased markedly. CA, carbohydrate antigen; CEA, carcinoembryonic antigen; Nivo, nivolumab; XELOX, oxaliplatin + capecitabine

is notorious for its aggressive nature, most investigators have suggested that these elements should be considered therapeutic targets (32). In 1999, Mitry et al (33) first reported the efficacy of etoposide and cisplatin regimens in a cohort of 53 neuroendocrine cancers (including 3 cases that occurred in gastric cancer); Uchiyama et al (2) introduced S-1-based regimens as adjuvant therapy for 7 cases, and the 3 -year overall survival rate was $83.8 \%$. Okita et al (34) reported that cisplatin plus irinotecan regimens received a good response in 12 cases. Notably, Ip et al (35) reported a spontaneous complete regression of gastric NEC that seemed to be mediated by cytomegalovirus-induced cross-autoimmunity. In our case, the patient was treated with the XELOX regimen based on the first pathological results, and whether replacement with schemes such as etoposide and cisplatin could have led to tumor regression is unknown because of the poor physical states and severe complications of the patient at the terminal stage.

In recent years, immunotherapy has become increasingly popular in treating cancers, but the efficacy of such therapies is still being validated. A major problem for such therapies is the lack of reliable biomarkers for patient selection and response evaluation. Predictive biomarkers such as PD-L1 were under extensive study to this end; unfortunately, although expression of PD-L1 was detected in 50\% of stage II and III gastric cancers (36), it has been found to be insufficient for patient selection for immunotherapy thus far (37). A phase $1 \mathrm{~b}$ clinical trial in 2016 first reported the application of pembrolizumab (another immunotherapy agent) for recurrent or metastatic gastric cancer (38). Subsequently, the efficacy of nivolumab in advanced gastric cancer was established in a phase 3 trial with a registered median overall survival of 5.26 months (39). Other agents targeting PD-L1 (such as avelumab) are still under clinical investigation. However, there are still no clinical trials concerning immunotherapeutic agents for concurrent or multiple cancers. In our case, the patient was treated with 


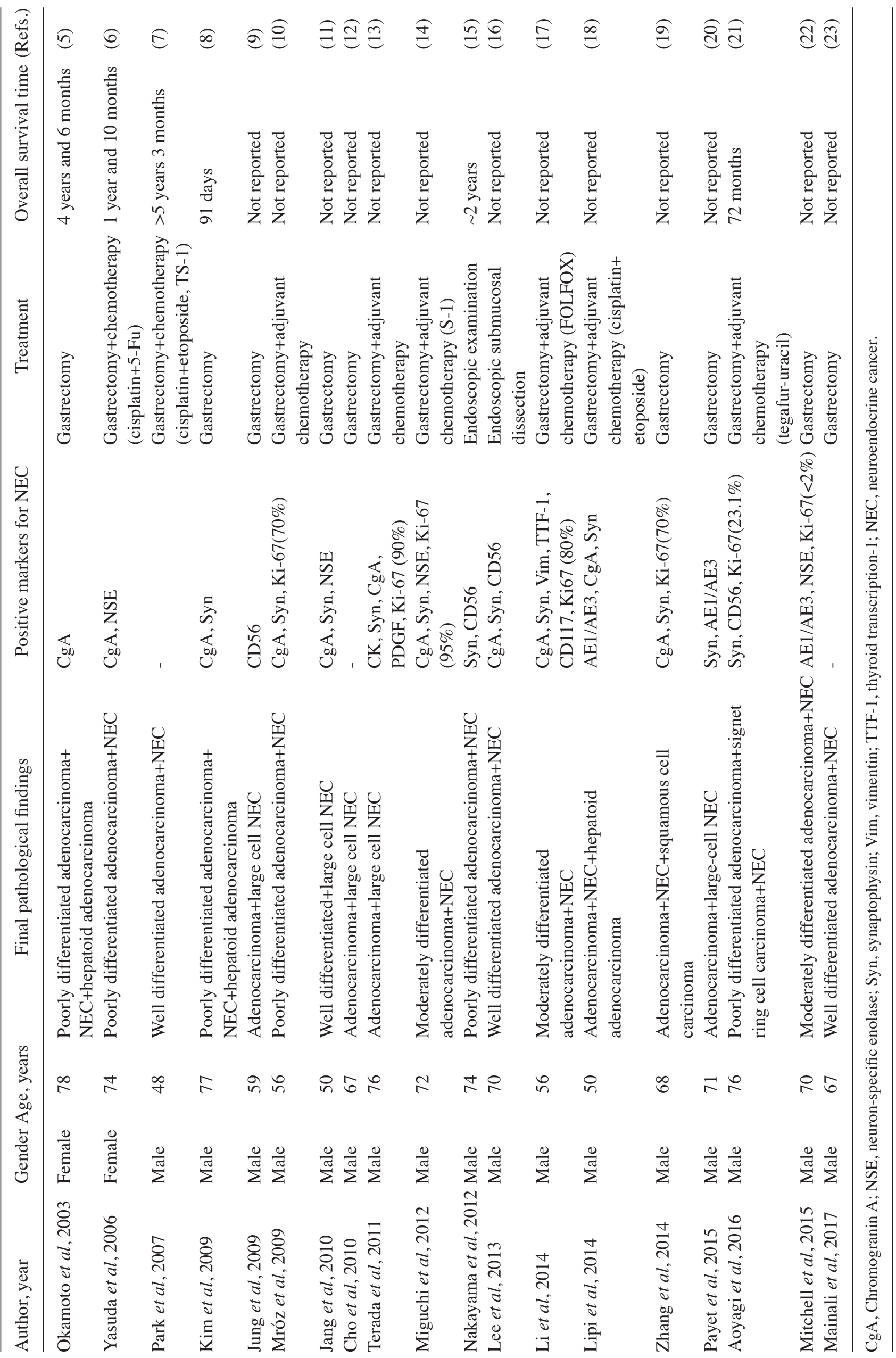




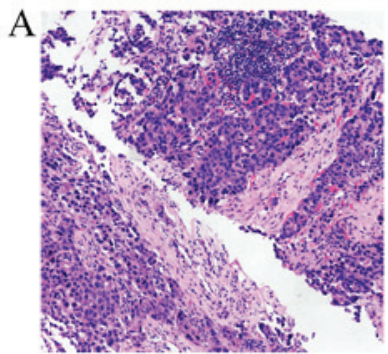

$\mathrm{E}$

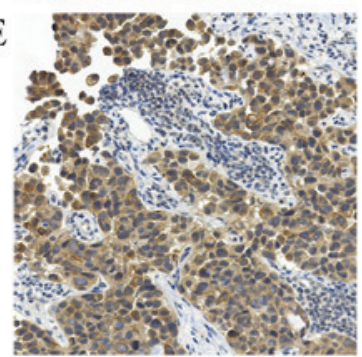

B

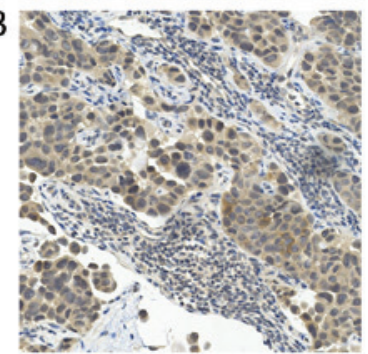

$\mathrm{F}$

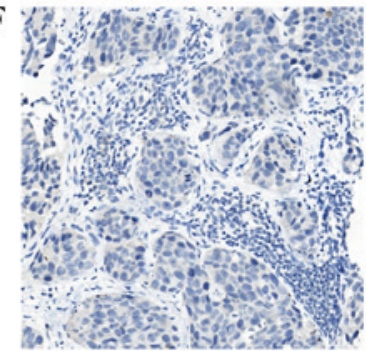

$\mathrm{C}$

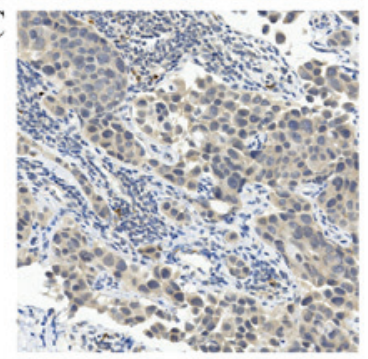

$\mathrm{G}$

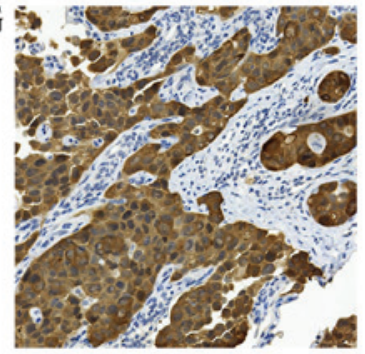

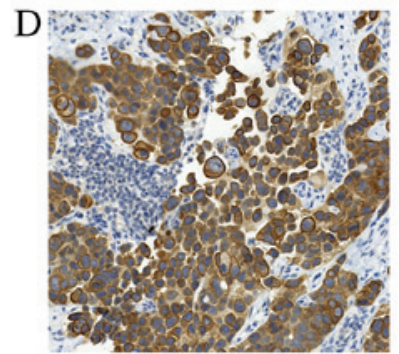

$\mathrm{H}$

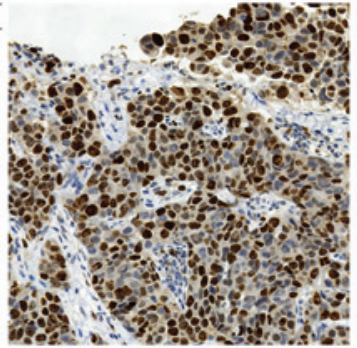

Figure 4. Histological results from biopsy specimens from metastatic collarbone lymph nodes (H\&E staining and immunohistochemistry). (A) Many poorly differentiated neuroendocrine cancer cells were clustered in the sample (H\&E: magnification, x10). (B-G) The cancer cells were diffusely positive for (B) Syn, (C) cluster of differentiation-56, (D) CK and (E) CK7, and were negative for (F) CK20 and (G) Vim (all, magnification, x20). (H) The cancer cells presented high proliferative capacity, and Ki-67 was diffusely positive with an index $>75 \%$ (magnification, x20). H\&E, hematoxylin and eosin; CK, creatine kniase; Syn, synaptophysin; Vim, vimentin.

the nivolumab plus XELOX regime; considering the reported PFS (5.8 months) and OS (11.8 months) of single XELOX in advanced gastric cancer (40), it is difficult to conclude whether such combined therapies could help prolong the OS for such patients; however, additional clinical studies are still needed in the future.

Concurrent gastric adenocarcinoma and NEC can occur in the clinic, and interpretation of the pathological results should be done cautiously in the absence of gross specimens. The efficacy of therapeutic strategies such as immunotherapy and chemotherapy still requires further validation.

\section{Acknowledgements}

Not applicable.

\section{Funding}

The present study was supported by the Natural Science Foundation of China (grant no. 81503391), Natural Science Foundation Project of Hainan (grant no. 817352) and Medical and Health Science Innovation Project of Sanya (grant no. 2016YW08).

\section{Availability of data and materials}

All data generated or analyzed during this study are included in this published article.

\section{Authors' contributions}

BY and MC performed the case study, collected the data and images of the case and produced the draft of the manuscript. JY, FL and HL critically analyzed the manuscript for important intellectual content. All authors read and approved the final manuscript.

\section{Ethics approval and consent to participate}

The present study was approved by the Medical Ethics Committee of the Hainan Branch of PLA General Hospital. Written informed consent was obtained from the patient's father.

\section{Patient consent for publication}

Written informed consent was obtained from the patient's father.

\section{Competing interests}

The authors declare that they have no competing interests.

\section{References}

1. Shah MA and Ajani JA: Gastric cancer-an enigmatic and heterogeneous disease. JAMA 303: 1753-1754, 2010.

2. Uchiyama C, Tamura S, Nakatsuka S, Takeno A, Miki H, Kanemura T, Nakahira S, Suzuki R, Nakata K, Takeda Y and Kato T: Immunohistochemical consistency between primary tumors and lymph node metastases of gastric neuroendocrine carcinoma. World J Surg Oncol 10: 115, 2012.

3. Fukui H, Takada M, Chiba T, Kashiwagi R, Sakane M, Tabata F, Kuroda Y, Ueda Y, Kawamata H, Imura J and Fujimori T: Concurrent occurrence of gastric adenocarcinoma and duodenal neuroendocrine cell carcinoma: A composite tumour or collision tumours? Gut 48: 853-856, 2001.

4. Kim BS, Park YS, Yook JH and Kim BS: Comparison of the prognostic values of the 2010 WHO classification, AJCC 7th edition and ENETS classification of gastric neuroendocrine tumors. Medicine (Baltimore) 95: e3977, 2016.

5. Okamoto T, Ogasahara K, Fujiki M, Takagi H, Ikeda H, Makino T, Moriga T, Kawamoto K, Sano K, Yoshida Y, et al: Primary coexistent neuroendocrine carcinoma, hepatoid adenocarcinoma, and tubular adenocarcinoma of the stomach with focal trophoblastic differentiation in metastatic lymph nodes. J Gastroenterol 38: 608-610, 2003.

6. Yasuda K, Fujiwara H, Nomura H, Sogo Y, Sakai K and Teramura K: A case of neuroendocrine cell carcinoma and poorly differentiated adenocarcinoma of the stomach in synchronous multiple cancer. Jpn J Gastroenterol Surg 39: 446-451, 2006. 
7. Park BS, Jo TY, Seo HI, Kim HS, Kim DH, Jeon TY, Kim DH, Sim MS and Kim JY: Gastric collision tumor (adenocarcinoma and neuroendocrine carcinoma) diagnosed as a advanced gastric cancer. J Korean Surg Soc 73: 173-177, 2007.

8. Kim A, Kim SW, Song SK and Bae YK: Gastric adenocarcinoma with coexistent hepatoid adenocarcinoma and neuroendocrine carcinoma: A case report. Korean J Pathol 43: 79-82, 2009.

9. Jung JH, Kim YC, Kim JH, Chung HS, Cheung DY, Kim JI, Park SH and Kim JK: A case of a gastric composite tumor with an adenocarcinoma and a large cell neuroendocrine carcinoma. Korean J Gastrointest Endosc 39: 93-96, 2009.

10. Mróz A, Kiedrowski M, Malinowska M and Sopyło R: Collision tumour of the stomach-adenocarcinoma and neuroendocrine carcinoma: Case report and review of the literature. Pol J Pathol 2: 94-97, 2009.

11. Jang KY, Moon WS, Lee H, Kim CY and Park HS: Gastric collision tumor of large cell neuroendocrine carcinoma and adenocarcinoma-a case report. Pathol Res Pract 206: 387-390, 2010.

12. Cho KH, Chang YW, Sohn SD, Hwangbo Y, Shim J, Jang JY, Kim HJ and Kim BH: Metastatic large cell neuroendocrine carcinoma combined with gastric adenocarcinoma. Korean J Gastrointest Endosc 41: 94-97, 2010.

13. Terada $\mathrm{T}$ and Maruo H: Simultaneous large cell neuroendocrine carcinoma and adenocarcinoma of the stomach. World $J$ Gastroenterol 17: 4831-4834, 2011.

14. Miguchi M, Iseki M and Shimatani K: Advanced gastric neuroendocrine carcinoma with an adenocarcinoma component. Case Rep Gastroenterol 6: 52-57, 2012.

15. Nakayama Y, Higure A, Shibao K, Sato N, Matayoshi N and Yamaguchi K: Synchronous occurrence of early neuroendocrine carcinoma and tubular adenocarcinoma in the stomach. Clin J Gastroenterol 5: 307-311, 2012.

16. Lee JH, Kim HW, Kang DH, Choi CW, Park SB and Kim SH: A gastric composite tumor with an adenocarcinoma and a neuroendocrine carcinoma: A case report. Clin Endosc 46: 280-283, 2013.

17. Li S, Cao X, Jiang C and Wang Q: Combined neuroendocrine carcinoma and adenocarcinoma in the stomach: A case report Oncol Lett 7: 953-955, 2014.

18. Lipi L, Sachdev R, Gautam D, Singh J and Mohapatra I: Triple composite tumor of stomach: A rare combination of alpha fetoprotein positive hepatoid adenocarcinoma, tubular adenocarcinoma and large cell neuroendocrine carcinoma. Indian J Pathol Microbiol 57: 98-100, 2014

19. Zhang W, Xiao W, Ma H, Sun M, Chen H and Zheng S: Neuroendocrine liver metastasis in gastric mixed adenoneuroendocrine carcinoma with trilineage cell differentiation: A case report. Int J Clin Exp Pathol 7: 6333-6338, 2014.

20. Payet E, Pilco PI, Montes J, Cordero-Morales A, Savitzky MJ and Stenning-Persivale K: Collision tumour of large-cell neuroendocrine carcinoma and adenocarcinoma in the stomach: A case report. Ecancermedicalscience 10: 616, 2016.

21. Aoyagi K, Kizaki J, Isobe T and Akaqi Y: A case of gastric cancer with neuroendocrine carcinoma, signet ring cell carcinoma components, and intramural metastases. Am J Case Rep 17: 274-279, 2016

22. Mitchell MS, Walinchus-Foster L, Thomas L, Shi Q, Nagori M, Khan W, Mirza W, Cortes M, Masalmeh OA, Zerti H and Mohamed A: Synchronous adenocarcinoma and neuroendocrine tumors of the stomach. Am J Cancer Case Rep 4: 125-128, 2016

23. Mainali N, Nepal N, Kumar CP, Sinha A, Rajbanshi S, Rauniyar S and Yadav A: Co-existence of gastric adenocarcinoma and neuroendocrine carcinoma: A rare entity. J Pathol Nepal 7: 1221-1223, 2017

24. Nishikura K, Watanabe H, Iwafuchi M, Fujiwara T, Kojima K and Ajioka Y: Carcinogenesis of gastric endocrine cell carcinoma: Analysis of histopathology and p53 gene alteration. Gastric Cancer 6: 203-209, 2003.

25. Kim JJ, Kim JY, Hur H, Cho YK and Han SU: Clinicopathologic significance of gastric adenocarcinoma with neuroendocrine features. J Gastric Cancer 11: 195-199, 2011.
26. Zhang T, Su D, Mao Z, Guo X, Wang L and Bai L: Prognostic role of neuroendocrine cell differentiation in human gastric carcinoma. Int J Clin Exp Med 8: 7837-7842, 2015.

27. Bakkelund K, Fossmark R, Nordrum I and Waldum H: Signet ring cells in gastric carcinomas are derived from neuroendocrine cells. J Histochem Cytochem 54: 615-621, 2006.

28. Yamazaki K: A gastric carcinosarcoma with neuroendocrine cell differentiation and undifferentiated spindle-shaped sarcoma component possibly progressing from the conventional tubular adenocarcinoma; an immunohistochemical and ultrastructural study. Virchows Arch 442: 77-81, 2003.

29. Lim SU, Seo SS, Yoon JY, Cho DH, Bae SY, Bae WK, Shim HJ, Cho SH and Chung IJ: A case of gastric adenocarcinoma developed in neuroendocrine carcinoma after chemotherapy. Chonnam Med J 44: 188-193, 2008.

30. La Rosa S, Marando A, Sessa F and Capella C: Mixed adenoneuroendocrine carcinomas (MANECs) of the gastrointestinal tract: An update. Cancers (Basel) 4: 11-30, 2012.

31. Sato Y, Hashimoto S, Mizuno K, Takeuchi M and Terai S Management of gastric and duodenal neuroendocrine tumors. World J Gastroenterol 22: 6817-6828, 2016.

32. Fukuda T, Ohnishi Y, Nishimaki T, Ohtani H and Tachikawa S: Early gastric cancer of the small cell type. Am J Gastroenterol 83: 1176-1179, 1988

33. Mitry E, Baudin E, Ducreux M, Sabourin JC, Rufié P, Aparicio T, Aparicio T, Lasser P, Elias D, Duvillard P, et al: Treatment of poorly differentiated neuroendocrine tumours with etoposide and cisplatin. Br J Cancer 81: 1351-1355, 1999.

34. Okita NT, Kato K, Takahari D, Hirashima Y, Nakajima TE, Matsubara J, Hamaguchi T, Yamada Y, Shimada Y, Taniguchi H and Shirao K: Neuroendocrine tumors of the stomach: Chemotherapy with cisplatin plus irinotecan is effective for gastric poorly-differentiated neuroendocrine carcinoma. Gastric Cancer 14: 161-165, 2011.

35. Ip YT, Pong WM, Kao SS and Chan JK: Spontaneous complete regression of gastric large-cell neuroendocrine carcinoma: Mediated by cytomegalovirus-induced cross-autoimmunity? Int J Surg Pathol 19: 355-358, 2011.

36. Zhang L, Qiu M, Jin Y, Ji J, Li B, Wang X, Yan S, Xu R and Yang D: Programmed cell death ligand 1 (PD-L1) expression on gastric cancer and its relationship with clinicopathologic factors. Int J Clin Exp Pathol 8: 11084-11091, 2015.

37. Gibney GT, Weiner LM and Atkins MB: Predictive biomarkers for checkpoint inhibitor-based immunotherapy. Lancet Oncol 17: e542-e551, 2016.

38. Muro K, Chung HC, Shankaran V, Geva R, Catenacci D, Gupta S, Eder JP, Golan T, Le DT, Burtness B, et al: Pembrolizumab for patients with PD-L1-positive advanced gastric cancer (KEYNOTE-012): A multicentre, open-label, phase 1b trial. Lancet Oncol 17: 717-726, 2016.

39. Kang YK, Boku N, Satoh T, Ryu MH, Chao Y, Kato K, Chung HC, Chen JS, Muro K, Kang WK, et al: Nivolumab in patients with advanced gastric or gastro-oesophageal junction cancer refractory to, or intolerant of, at least two previous chemotherapy regimens (ONO-4538-12, ATTRACTION-2): A randomised, double-blind, placebo-controlled, phase 3 trial. Lancet 390: 2461-2471, 2017.

40. Park YH, Lee JL, Ryoo BY, Ryu MH, Yang SH, Kim BS, Shin DB, Chang HM, Kim TW, Yuh YJ and Kang YK: Capecitabine in combination with Oxaliplatin (XELOX) as a first-line therapy for advanced gastric cancer. Cancer Chemother Pharmacol 61: 623-629, 2008

This work is licensed under a Creative Commons Attribution-NonCommercial-NoDerivatives 4.0 International (CC BY-NC-ND 4.0) License. 